\title{
Horner Syndrome Secondary to Central Venous Catheter
}

\author{
Shaza Aouthmany MD and Alexandra Lu MD \\ Department of Emergency Medicine at the University of Toledo College of Medicine and Life Sciences
}

Summary

*Corresponding author: Shaza Aouthmany, Department of Emergency Medicine at the University of Toledo College of Medicine and Life Sciences, USA

\section{Summary}

64 years old female admitted to the hospital for GI bleed, developed hypotension and required a central venous catheter. After 4 unsuccessful attempts of placing a central line, the patient immediately developed ptosis of the right eye, with associated anisocoria. Patient as transferred to the ICU due to hemodynamic instability and for further work up. Neurology was consulted and CT brain non contrast as well as CTA carotids were ordered. CT brain was unremarkable and CTA carotids showed all neck vasculature to be widely patent with no hematomas or other acute abnormalities noted on the imaging. A diagnosis of horner syndrome secondary to right IJ central venous access was made.

\section{Background}

Horner Syndrome secondary to central venous catheter (CVC) is a relatively uncommon complication of the procedure, however it is a complication that should be considered when placing CVC. Care should be taken during the procedure to avoid injury to the carotid sheath. If injury is suspected, further workup is needed to determine severity. Horner's syndrome associated with CVC of the IJ is caused by the damage to the nerve plexus that lies adjacent to the carotid artery sheath.

\section{Case Presentation}

A 64-year-old female, with past medical history of Multiple Sclerosis, presented to an emergency department for hematemesis and weakness. Upon presentation to the emergency department, the patient had one episode of hematemesis as well as a positive stool guiac. Patient's initial labs showed a stable hemoglobin of 9.0, normal coagulation profile and a CT abdomen that showed nonspecific wall thickening of the gastroduodenal junction. The patient remained hemodynamically stable within the emergency department, received intravenous (IV) fluids and IV pepcid and was admitted to the hospital for further workup of the gastrointestinal (GI) bleed.

While in the hospital, the patient had a bloody bowel movement which caused her to become hypotensive with systolic pressure in the 50s and was minimally responsive.
The decision was made to place a central line for volume resuscitation/ possible pressor support. A right IJ triple lumen CVC was attempted using ultrasound to guide venous access. 4 attempts to thread the guidewire in the internal jugular (IJ) were unsuccessful and the procedure was terminated. The patient states that immediately following the failed IJ central line procedure, she started experiencing ptosis of the right eye, with associated anisocoria (minimal--1 $\mathrm{mm}$ difference between the eyes).

Following the failed attempt at right IJ CVC, the decision was made to transfer the patient to a hospital with higher capabilities due to her hemodynamic instability. Upon presentation to the hospital, the patient was transferred to the ICU. She was evaluated by neurology and received CT brain without contrast as well as CTA of the carotids. CT brain was unremarkable and CTA carotids showed all neck vasculature to be widely patent with no hematomas or other acute abnormalities noted on the imaging. MS exacerbation was considered as a source of the patient's presentation but the timing of the symptoms in relation to the procedure made this an unlikely cause of the patient's pathology. A diagnosis of horner syndrome secondary to right IJ central venous access was made.

\section{Discussion}

Horner's syndrome is a classic neurological syndrome characterized by ipsilateral blepharoptosis, pupillary miosis, 


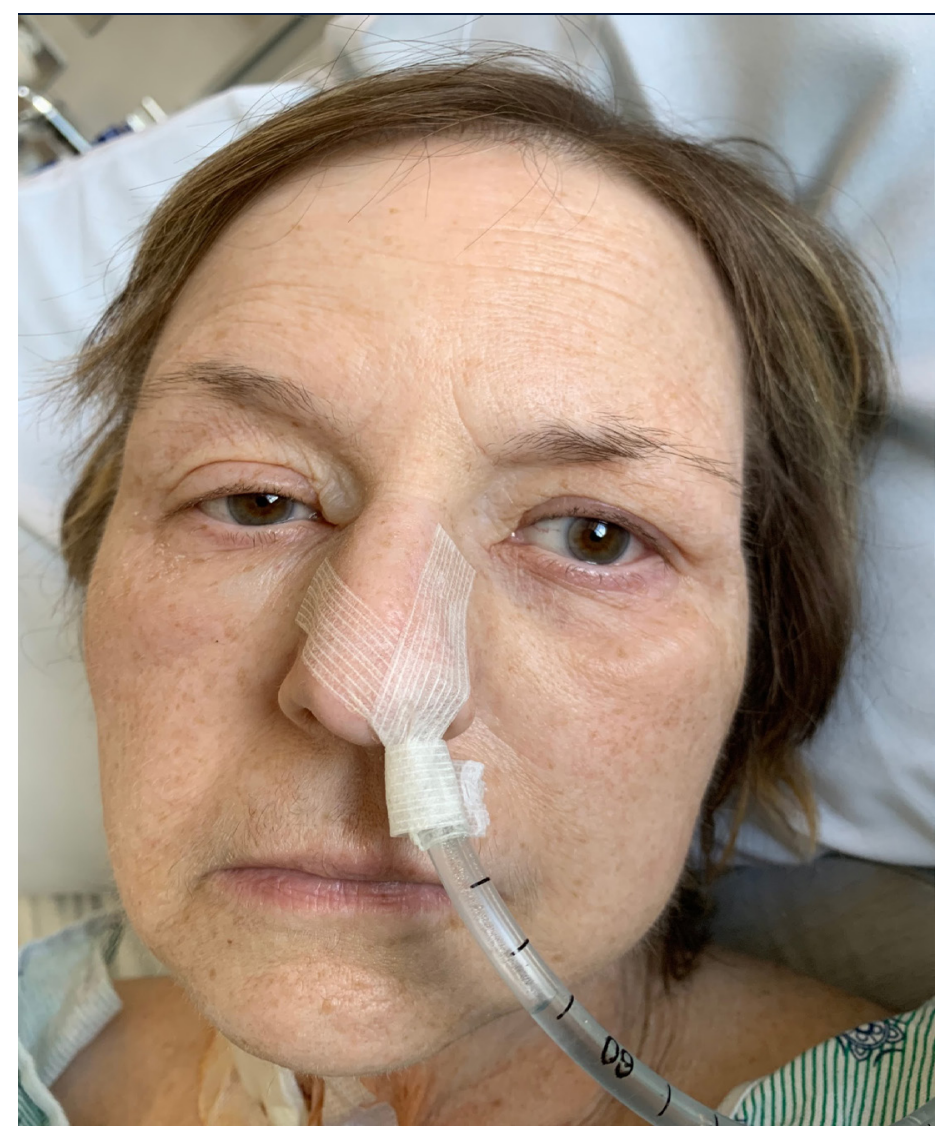

Figure 1

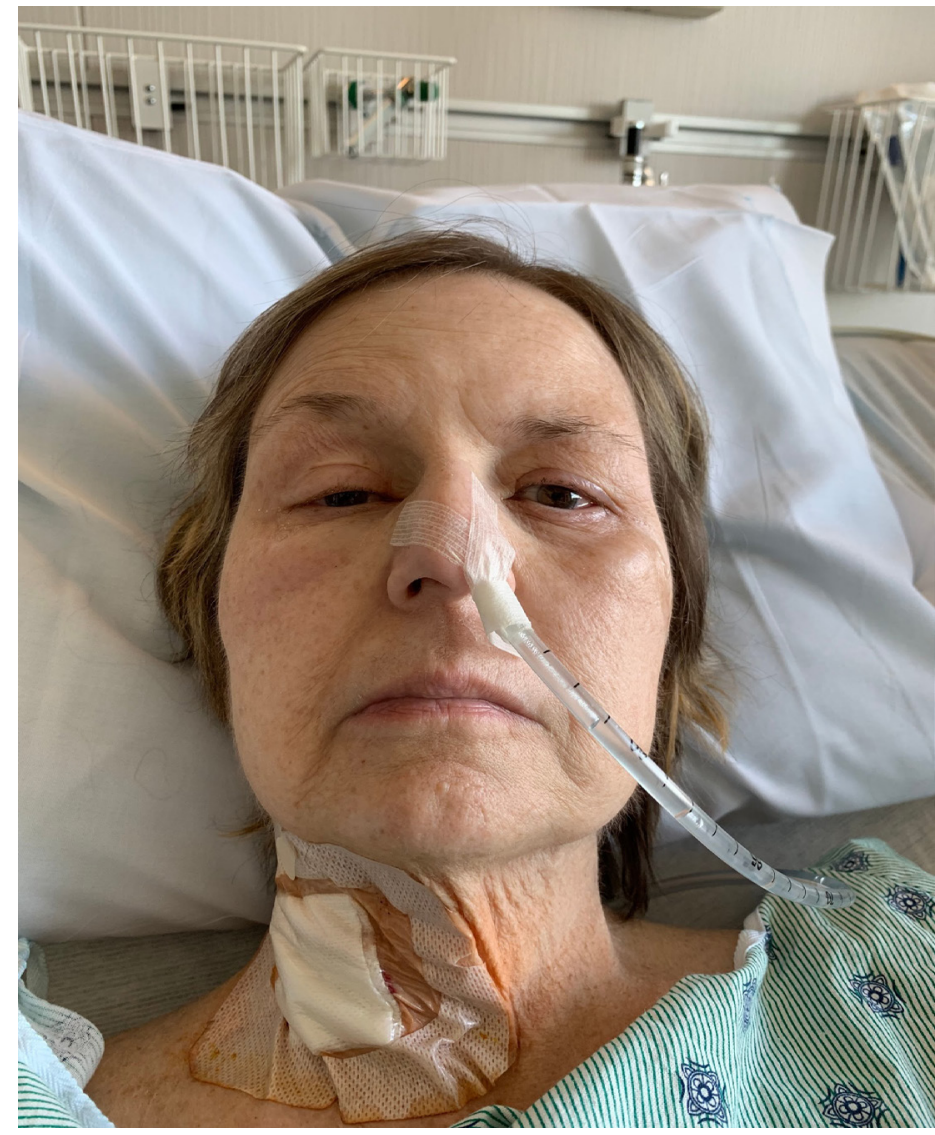

Figure 2 and facial anhidrosis [1]. It should be noted that the diagnosis of Horner's syndrome can be made without the existence of all three diagnostic criteria. Depending on where the level of injury to the nerves occurs, the presentation of Horner syndrome will differ. The sympathetic nerves that innervate the areas involved in Horner syndrome arise in the hypothalamus, run down the cervical spine, along the brachial plexus, around the internal carotid sheath and finally run-in close relation to the respective cranial nerves [2]. The lengthy course of the sympathetic nerve fibers makes them particularly susceptible to injury.

In the instance of CVC in the IJ, injury to nerve fibers occurs in the post-ganglionic region. The post-ganglionic region contains nerves responsible for innervating the pupil and palpebral muscles. Injury in this area will present with anisocoria and ptosis [3]. Injury at the level of the postganglionic nerves is marked by lack of facial anhidrosis. The nerves responsible for facial sweating arise earlier in the pathway and are relatively sparse at the level of the carotid sheath around the IJ [3].

Horner's syndrome is an uncommon complication of central venous catheter insertion (CVC) occurring in around $2 \%$ of CVCs [4]. Horner's syndrome associated with CVC of the IJ is caused by the damage to the nerve plexus that lies adjacent to the carotid artery sheath [4]. Because of the nerve's location in the carotid sheath, they are prone to damage if trauma to the area surrounding the carotid artery occurs. In the instance of IJ CVC, this damage occurs via expanding hematoma or direct injury by misguided CVC insertion (particularly if there are multiple attempts at insertion-- as was the case with this patient) [5]. If Horner syndrome secondary to CVC insertion is suspected, workup should be performed to ensure the carotid artery has not been critically damaged. If this is the case, further intervention may be required. Without significant carotid injury, Horner syndrome related to CVC can be expected to improve or resolve over the following weeks/months.

\section{Learning Points}

1. Horner's syndrome secondary to central venous catheter is rare.

2. Further workup is critical if Horner's syndrome secondary to CVC develops to ensure carotid artery is not damaged.

3. Without significant injury to the carotid artery, Horner syndrome secondary to CVC, is expected to improve or resolve within weeks to months.

4. Ultrasound guidance can be a valuable tool to

Citation: Shaza Aouthmany MD and Alexandra Lu MD .Horner Syndrome Secondary to Central Venous Catheter Op Acc J Bio Sci \& Res 7(1)-2021. 
assist the clinician in preventing unnecessary trauma to the carotid artery precipitating central venous catheter related Horner syndrome.

\section{References}

1. Kardon R (2005) Anatomy and physiology of the autonomic nervous system. In: Walsh and Hoyt Clinical Neuro-ophthalmology, 6th ed, Miller NR, Newman NJ, Biousse V, Kerrison JB (Eds), Williams \& Wilkins, Baltimore pp. 649.
2. Khan Z, Bollu PC (2019) Horner Syndrome. In: StatPearls [Internet]. Treasure Island (FL): StatPearls Publishing, USA.

3. Kanagalingam S, Miller NR (2015) Horner syndrome: clinical perspectives. Eye Brain 7: 35-46.

4. Butty Z, Gopwani J, Mehta S, Margolin E (2016) Horner's syndrome in patients admitted to the intensive care unit that have undergone central venous catheterization: a prospective study. Eye (Lond) 30(1): 31-33.

5. Vinod KV, Sivabal V, Vidya MV (2017) Iatrogenic Horner's syndrome: A cause for diagnostic confusion in the emergency department. World J Emerg Med 8(3): 235-236.

*Corresponding author: Shaza Aouthmany, Email: shaza.aouthmany@utoledo.edu

Next Submission with BGSR follows:

- Rapid Peer Review

- Reprints for Original Copy

- $\quad$ E-Prints Availability

- Below URL for auxiliary Submission Link: https://biogenericpublishers.com/submit-manuscript/ 